\title{
Elaboração de Programas de Treinamento de Força para Crianças
}

\author{
Development of Weigh Training Programs for Children
}

\author{
Arli Ramos de Oliveira ${ }^{1}$; Andrei Guilherme Lopes ${ }^{2}$; Sidiclei Risso ${ }^{3}$
}

\begin{abstract}
Resumo
Um estilo de vida hipocinético tem aumentado entre crianças e adolescentes nos últimos anos, e isso faz com que os fatores de risco se apresentem mais facilmente, favorecendo o surgimento de doenças crônico-degenerativas já na infância. Logo, torna-se necessário a criação de programas de atividade física para essa faixa etária. O objetivo desse estudo é realizar uma revisão de literatura acerca dos fatores que se relacionam à montagem de um programa de treinamento com sobrecarga para crianças. Discutemse aspectos relacionados à atividade física na infância, gasto calórico, aptidão física relacionada à saúde, motivos que levam as crianças à praticar, ou desistir da atividade física, estabilidade da aptidão física na infância, aspectos fisiológicos e fatores de crescimento. Mais específicamente, são tratados os fatores que interferem na capacidade motora força, os mitos relacionados ao treinamento de força muscular em crianças, possíveis benefícios advindos dessa prática, fatores que devem ser levados em consideração no treinamento de força específico para crianças, supervisão do treinamento de força muscular em crianças, orientações para progressão do treinamento de força muscular em crianças, elaboração dos programas de treinamento de força muscular para crianças e adolescentes e discussão acerca dos testes de força. Quando devidamente supervisionado por especialistas, o treinamento específico e individualizado para crianças e adolescentes pode propiciar um ganho considerável de força muscular, e contribuir favoravelmente para a promoção da saúde e qualidade de vida nessa faixa etária.
\end{abstract}

Palavras-chave: Treinamento com sobrecarga. Força muscular. Crianças.

\begin{abstract}
A hypokinetic lifestyle has increased among children and adolescents in the past few years, leading to risk factors which favor the chronic-degenerative diseases in the early childhood. Thus, the development of physical activity programs for this age group becomes necessary. The purpose of this study was to review the literature about the factors that can be related to the development of weight training programs with extra load for children. Aspects related to the physical activity in the childhood, caloric damage, physical fitness related to health, reasons that make children and adolescents practise or give up physical activity, physical fitness stability during childhood, physiological aspects and growth factors, are discussed. The factors that can interfere in the motor capacity strength, the myths related to weight training with children, possible benefits obtained from this practice, factors that should be taken into consideration regarding specific weight training for children, weight training supervision during childhood, general guidelines for weight training in children, developing weight training programs for children and discussion about strength testing, are dealt with more specifically. When supervised properly by specialists, the specific and individualized training for children and adolescents can generate a significant muscular strength gain, and contribute favorably for health promotion and life quality in this age group. Key words: Weight training. Muscular strength. Children.
\end{abstract}

\footnotetext{
Professor Adjunto no Curso de Licenciatura em Educação Física da Universidade Estadual de Londrina e nos Cursos de Especialização em Treinamento Desportivo, Educação Física no Ensino Básico, Lazer e Hospitalidade. E-mail: arli@uel.br

2 Membro da Comissão de Pesquisa do Departamento de Ginástica, Recreação e Dança do Centro de Educação Física e Desportos.

3 Membro do Comitê de Ética em Pesquisa da Universidade Estadual de Londrina.
} 


\section{Introdução}

No passado, as crianças eram mais ativas fisicamente, mas em função de uma vida moderna, elas têm adotado uma postura hipocinética. $\mathrm{Na}$ infância e adolescência, há necessidade de se utilizar os exercícios físicos como prevenção aos fatores de risco que podem influenciar a saúde de cada indivíduo. As doenças crônico-degenerativas são causadas por fatores de risco, que, por sua vez, originam-se dos maus hábitos. Esse quadro porém, pode ser revertido através da atividade física regular, controle alimentar e controle do estresse.

A prática de atividade física deve ser iniciada já na infância, de acordo com as condições relativas ao estágio de desenvolvimento em que a criança se encontra. Ela é um pré-requisito para o seu crescimento e desenvolvimento, e, dessa forma, aumentará a probabilidade do indivíduo assumir um comportamento físico ativo quando adulto. O estado de morbidez deve ser afastado ao máximo sob o risco de distúrbios orgânicos irreversíveis na vida adulta. A criança poderá maximizar os benefícios se estiver disposta a realizar a atividade.

Distúrbios no controle do peso corporal originamse geralmente na infância, e desse fato decorre a possibilidade de se ter um adulto obeso. Esse quadro é decorrente de um baixo nível de atividade física e de um controle alimentar ineficiente.

Torna-se necessária a investigação acerca da elaboração de programas de treinamento de força para crianças, já que esta capacidade física é um dos componentes da aptidão física relacionada à saúde.

Logo, o objetivo deste estudo é investigar, através de uma revisão de literatura, a influência da atividade física no desenvolvimento da criança, especialmente na realização de exercícios com sobrecarga.

Buscam-se, neste estudo, esclarecimentos acerca do treinamento de força muscular em crianças, seus benefícios, aspectos de segurança e auxílio, princípios básicos do treinamento de força, diferentes faixas etárias, princípios metodológicos, tipos de testes, didática do treinamento e aspectos fisiológicos.

\section{Aspectos Relacionados à Atividade Física em Crianças}

$\mathrm{O}$ mundo atual proporciona à criança uma diminuição dos esforços físicos e adução de maus hábitos alimentares, o que provoca um desequilíbrio calórico, levando geralmente a criança a um estado de sobrepeso (e até de obesidade) muito precoce. Crianças ativas fisicamente apresentam benefícios fisiológicos no sistema cardiovascular. O conhecimento da prática da atividade física por ela realizada leva-a a tornar-se mais ativo. Os pais são indispensáveis no controle da alimentação, atividade física e possíveis complicações fisiológicas nas crianças. Os pais também podem contribuir no estilo de vida que seus filhos terão. Pais mais ativos fisicamente e ambiente favorável à prática de atividade física possibilitam que crianças e adolescentes adquiram mais facilmente este hábito. Os alimentos consumidos constituem o combustível para o trabalho biológico juntamente com o oxigênio, e desse processo resulta a energia necessária para as atividades físicas e funcionais indispensáveis à vida. A energia química dos alimentos é transformada em energia mecânica. $\mathrm{O}$ gasto e a ingestão de energia varia de indivíduo para indivíduo, dependendo do tamanho do corpo, eficiência mecânica e atividade física. $\mathrm{O}$ gasto energético das crianças depende do metabolismo basal, velocidade de crescimento, tamanho corporal, idade e nível de atividade física. Alguns métodos utilizados para determinar o nível de atividade física e/ou gasto energético são: diário de atividades, observação direta, questionários, sensores eletrônicos de movimento e monitor de freqüência cardíaca. (PINHO; PETROSKI, 1997).

Um estudo realizado em escolares no Município de Londrina, no Estado do Paraná, indicou que somente quinze por cento das crianças e adolescentes atingiram as exigências motoras mínimas estabelecidas pela literatura na tentativa de satisfazer 
aos aspectos relacionados à saúde, reduzindo-se esta porcentagem após os onze anos de idade. Esses resultados demonstraram índices de desempenho motor e quantidade de gordura corporal que podem comprometer a obtenção de um melhor nível de qualidade de vida, sugerindo a implementação de programas de atividades físicas para crianças e adolescentes. A melhora no desempenho motor e na quantidade de gordura corporal podem provocar melhoras na condição de saúde com repercussão para toda a vida (GUEDES; GUEDES, 1995).

Num estudo realizado por Brito et al. (1999) com crianças e adolescentes de 09 a 15 anos de idade aproximadamente, seguidas longitudinalmente, apresentaram um nível de estabilidade variando de moderado a alto nas variáveis motoras (com exceção do VO2 max que demonstra instabilidade durante esse período da vida) e antropométricas, com estas aparentando mais estabilidade.

Segundo Malina (1996 apud BRITO et al., 1999), o baixo nível de estabilidade de algumas variáveis neuromotoras durante a infância, provavelmente refletem a variação do amadurecimento dos padrões de movimento, a individualidade do ritmo e progresso de crescimento. $\mathrm{O}$ diagnóstico precoce da aptidão física pode facilitar a melhora na elaboração de programas de manutenção e melhoria da aptidão física para a promoção da saúde.

Os principais motivos que levam crianças a praticar atividade física são: melhorar suas habilidades físicas, estar com os amigos, divertir-se, ter gosto pelo desafio, fazer novas amizades, conquistar o sucesso e desenvolver a aptidão física. A atividade física, se desenvolvida baseada nos princípios biológicos, proporciona aumento no desempenho motor e benefícios psicológicos, levando sempre em consideração o nível maturacional da criança. É importante estimular a atividade física regular com participação do indivíduo durante toda a vida, visando saúde e bem estar. Práticas esportivas competitivas podem influenciar positivamente no aparecimento de lesões, mas a prática das modalidades esportivas pode ser um ótimo instrumento de motivação para que crianças e adolescentes possuam um ambiente ativo fisicamente e aumentem seu gasto calórico, conseguindo, dessa forma, também aumentar suas atividades metabólicas. Crianças tendem a cessar a prática de atividade física por não possuírem tempo para participação nas atividades, ênfase excessiva na competição, tédio, falta de prazer na atividade física e/ ou aversão ao treinador. Os objetivos e interesses individuais devem ser alcançados. Se a atividade física não proporcionar prazer ou bem-estar para a criança, certamente ela irá desistir de sua prática Aspectos genéticos, ambientais e psicossociais devem ser considerados na criança praticante de atividade física. Deve-se também respeitar as diferenças individuais e culturais. Crianças que possuem pais ativos tendem a ser mais ativas fisicamente. Crianças com menos de dez anos de idade analisam o resultado de cada atividade, crianças mais velhas e pré-adolescente se orientam mais pela avaliação de seus companheiros e comparações com pontos para julgar seu desempenho e competência. Um estilo de vida saudável, envolve sono adequado, controle do peso corporal, evitar fumo e álcool, alimentação adequada e prática regular de atividade física (OLIVEIRA, 1996).

O crescimento não se dá de forma contínua, e os segmento esqueléticos possuem diferentes épocas de desenvolvimento. Na puberdade, ocorre a aceleração do crescimento, aumento nos níveis de testosterona, diferenciação nas fibras lentas e rápidas, diferenciação entre os sexos (antropometria e massa muscular) e menarca (início da função menstrual). O metabolismo basal nas crianças é vinte porcento maior, quando comparado ao dos adultos. $\mathrm{O}$ excesso de atividade física contribui de forma negativa para o crescimento mas, uma programação com treinamento físico e controle alimentar pode contribuir para o desenvolvimento pleno da potencialidade geneticamente determinada. A sensibilidade dos tecidos é proporcional à sua velocidade de envelhecimento. $\mathrm{O}$ treinamento proporciona uma maior liberação do hormônio do crescimento, diminuindo com o passar dos anos. O hormônio do 
crescimento aumenta a síntese protéica, a mobilização dos ácidos graxos do tecido adiposo, diminui a utilização de glicose no organismo e estimula o crescimento ósseo (maior acúmulo de cálcio). A atividade física estimula o crescimento longitudinal e o crescimento em espessura dos ossos, melhora o controle do peso corporal, aumenta a força muscular e a flexibilidade, proporciona maior resistência cardiorrespiratória, baixando os níveis de colesterol e triglicerídeos no plasma, prevenindo doenças cardiocirculatórias (RAMOS, 1998).

A atividade física mostra-se indispensável para o desenvolvimento motor e para um bom nível de atividade física relacionada à saúde, devendo, dessa forma, abranger todos os componentes da mesma. Deste modo, trataremos à seguir, especificamente sobre o treinamento de força em crianças.

\section{Treinamento de Força Muscular em Crianças}

A força é uma qualidade que permite ao músculo ou grupo de músculos vencer uma resistência ao movimento do qual ele é o agente motor. Diversos fatores podem influenciar o desenvolvimento da força: sistema nervoso, raça, sexo, tipo de fibra, freqüência de sessões de treinamento, alimentação entre outras (ROCHA, 1978). A força envolve basicamente realizar exercícios contra uma determinada resistência, que visa condicionar uma resposta fisiológica corporal para certa atividade a ser realizada, podendo ser recreativa ou específica, com um objetivo a ser atingido (FLECK,1997).

A força muscular, há muito tempo, vem sendo considerada um importante componente da aptidão física. As pesquisas na musculação em crianças têm investigado se o treinamento pode trazer benefícios ou não para a sua saúde, crescimento e desenvolvimento. Alguns pesquisadores se opõem a esse tipo de treinamento, nessa faixa etária, com argumentos como a falta de hormônios específicos para aumentar a força muscular, falha no sentido de diminuir o risco de lesões em outras atividades, e a probabilidade de que este treinamento possa impedir o crescimento normal da criança. O treinamento de força em grupos do sexo masculino, feminino e combinado nas diferentes faixas etárias evidenciaram que o grupo pré-adolescente pode obter melhor desempenho, porém, os resultados necessitam de maior clareza. $\mathrm{O}$ resultado do treinamento tende a variar de acordo com a motivação dos participantes (OLIVEIRA; GALLAGHER; SILVA, 1995).

A criança parece não apresentar condições favoráveis ao desporto de rendimento antes do fim da puberdade, podendo levá-la a desistência da prática da modalidade. Sua força muscular pode ser efetivamente melhorada à custa dos componentes coordenativos e de ativação neuromuscular. $\mathrm{O}$ treinamento de força pode contribuir para a proteção do aparelho locomotor, reforçando-o e robustecendoo. Devem ser observados cuidados especiais com a coluna vertebral, principalmente antes da puberdade. A carga de treinamento deverá ser inferior à carga máxima, caso contrário, poderá haver repercussão no crescimento, comprometendo o tecido ósseo.

O treinamento deverá respeitar as particularidades do crescimento da criança. Os possíveis benefícios advindos do treinamento são, entre outros: Prevenção e correção de deficiências posturais; estimulação biológica favorável ao crescimento e desenvolvimento; aquisição de novas e mais complexas atividades técnicas relacionadas às anteriormente desenvolvidas (RISSO; LOPES; OLIVEIRA, 1999).

Os cuidados com a sobrecarga se fazem necessários ao relacionar o treinamento com o crescimento ósseo, pois este ainda não se encontra em sua formação final. Crianças e jovens que praticam atividade física sem controle de carga podem sofrer microtraumatismos na junção das unidades músculo-tendinosas ao osso. Durante esse período, músculos, tendões e ligamentos são de duas a cinco vezes mais fortes que suas inserções nos ossos, podendo resultar em inflamação ou lesão. Deve-se evitar: a técnica incorreta na realização dos exercícios (má execução motora e/ou excesso de 
sobrecarga para um determinado número de repetições); a ansiedade em querer fazer muito em pouco tempo, aumentando subitamente a intensidade, não respeitando a individualidade biológica; a especialização precoce, resultando em estresse mecânico sobre as estruturas músculo-tendinosas, ligamentosas e ósseas.

Lopes (2003) investigou as possíveis alterações epifisiárias ocorridas em função do treinamento de força muscular em pré-púberes, avaliadas por pilosidade púbica. As crianças foram submetidas a um período de 4 semanas de adaptação ao treinamento, e a 12 semanas de treino com sobrecarga a $80 \%$ do Teste de 1-RM proposto por Roberts e Weider (1994). Foi realizado exame radiológico nas articulações do cotovelo e joelho direito. Após o período de treinamento, as crianças repetiram as avaliações radiológicas seguindo os procedimentos iniciais, e os resultados indicaram não ter havido alterações das epífises dos ossos longos.

Um dos objetivos dos exercícios deve ser a manutenção e a construção de uma equilibrada estética corporal, com desenvolvimento harmonioso do corpo, com a função de prevenção e compensação de danos e lesões no aparelho de suporte e locomoção. Para isso, torna-se fundamental uma avaliação física prévia e avaliações posteriores para obtenção de dados que possam dar rumo ao treinamento.

O treinamento de força pode favorecer ou limitar o nível de outras capacidades físicas. Se o treinamento de base for adequado e bem conduzido, os riscos serão praticamente nulos. Alguns princípios metodológicos devem ser seguidos: a forma de trabalho deve ser compatível e atender o objetivo do praticante; ter planejamento a longo prazo, objetivando formação motora sem pensar em alto rendimento na idade infantil e juvenil; ter controle médico e avaliação constante para minimizar o risco de lesão ou danos.

A fim de manter o equilíbrio muscular, faz-se necessário realizar o aquecimento, que deve ser ativo e comportar as estruturas implicadas no movimento, sem deixar de ter caráter geral; executar corretamente os exercícios; realizar exercícios de alongamento, tanto na fase de aquecimento como na de relaxamento; proteger a coluna vertebral e articulações, priorizando exercícios na posição horizontal; fazer um rigoroso e sistemático controle médico-desportivo; controlar a intensidade e a carga, evitando maximizá-la; não desprezar exercícios de força estática, porém ter um controle da pressão arterial de todas as crianças; interromper o treinamento imediatamente após a sensação de dor .

O desenvolvimento inadequado da força tornará impossível a formação dos hábitos motores. $\mathrm{O}$ potencial de uma pessoa para a elaboração da força muscular é estabelecido ao nascer, pois cada um nasce com o número fixo de fibras musculares e este continua inalterado por toda a vida. $\mathrm{O}$ treinamento aumenta a força muscular assim como a inatividade a reduz. O desenvolvimento da força na prépuberdade não apresenta diferença entre os sexos.

Exercícios com sobrecarga adequada às possibilidades do organismo influem favoravelmente na constituição física e melhoram a capacidade dos órgãos e sistemas do organismo jovem (FIORESE, 1989).

Alguns preconceitos estão sendo derrubados em relação ao treinamento de força muscular em crianças (OLIVEIRA; GALLAGHER, 1999; OLIVEIRA; GALLAGHER, 1998; OLIVEIRA; GALLAGHER, 1997; OLIVEIRA; GALLAGHER, 1995), como o comprometimento do crescimento cartilaginoso, fraturas, lesões crônicas e problemas lombares. A esse respeito, tem-se verificado que, com uma adequada supervisão da sobrecarga, a incidência de lesão é praticamente nula. Os problemas lombares podem ocorrer devido à hiperlordose que tende a se desenvolver no período próximo à puberdade. Deve-se ter em mente, diante desse quadro, quais são as prioridades para cada criança previamente avaliada.

Alguns custos podem inviabilizar o treinamento de força em crianças tais como: equipamento, espaço 
e manutenção, custos nutricionais, médicos e psicológicos. A quantidade de exercícios para se atingir os objetivos consome tempo e envolve custos para gerar resultados. O treinamento deve ser supervisionado com a adaptação dos equipamentos e controle da intensidade, duração e volume. O supervisor deve estar atento ao nível de motivação e capacidade de atenção seletiva da criança. O prazer pela prática, a alegria e descontração devem ser valorizados nessa atividade. Um treinamento adequado pode proporcionar o aumento da força muscular, por meio de um maior recrutamento de unidades motoras, da ativação neuromuscular e da melhoria na coordenação motora; melhoria nos testes motores de aptidão física e performance; melhoria no desempenho esportivo e diminuição na ocorrência de lesões; aumento da força isométrica máxima em meninas entre nove e dez anos e meninos entre onze e treze anos (a força aumenta naturalmente e pode ser maximizada com o aumento da estatura em ambos os sexos); manutenção da aptidão física relacionada à saúde; redução do estresse emocional e do tempo de recuperação de lesões, auxiliando na prevenção de doenças músculoesqueléticas de longa duração; aumento da auto-estima, imagem e consciência corporal; melhora nas medidas de composição corporal; diminuição da pressão sangüínea em hipertensos; melhora nos níveis de lipídios no sangue e diminuição da quantidade de gordura corporal; aumento na densidade óssea; aumento do tempo de fadiga muscular e, consequentemente, diminuição da exaustão e lesões.

O treinamento deve ser elaborado e acompanhado por um profissional da atividade física capacitado para este tipo de treinamento. $\mathrm{O}$ destreinamento ocorre em adultos e em crianças e para a manutenção da força muscular, o treinamento deve ser mantido. Os tipos de testes para a determinação do nível da força muscular encontrados em alguns estudos são: isométrico, pesos livres, calistênico, isocinético, pneumático e hidráulico (RISSO; LOPES; OLIVEIRA, 1999).

As formas de treinamento mais utilizadas envolvem o peso do próprio corpo ou do companheiro, forma lúdica (jogos), sistematizada (musculação ou circuito) e pesos livres. Fleck (1997) sugere que o treinamento de força com crianças deve observar o prazer pela prática e a individualidade biológica, ter objetivo de promoção da saúde, propiciar vivência motora e conscientização quanto à prática de atividade física. Para a obtenção de bons resultados, é preciso utilizar técnicas adequadas, adaptar o equipamento utilizado, observar medidas de segurança, definir o acompanhamento constante de um profissional de atividade física, exame médico, orientar a postura correta na execução dos exercícios e utilizar a respiração de forma controlada inspirando na fase excêntrica e expirando na fase concêntrica.

Para a elaboração de um programa de treinamento de força sistematizado, sugere-se que haja equilíbrio entre os exercícios escolhidos, devendo haver ao menos um para cada grupo muscular. Observar a simetria na escolha dos exercícios para cada grupo muscular, selecionando exercícios que permitam o desenvolvimento de uma ou várias articulações. A sobrecarga utilizada deve permitir a execução de pelo menos seis repetições por série, o número de séries deve ser de uma a três. $O$ incremento da sobrecarga em cada exercício não deve ser superior a 5\% (cinco por cento) do peso utilizado para um determinado número de repetições, dependendo da idade da criança e da sua maturação psicológica. Cada sessão de treinamento deve ser realizada com duração entre vinte e sessenta minutos, devendo o treinamento ter a frequência de três vezes por semana. Deve-se utilizar, para a análise da sobrecarga, o Teste de Peso por Repetição Máxima (TPRM) (ROBERTS; WEIDER, 1994).

Em seguida trataremos de um método de treinamento de força e suas principais características.

\section{Treinamento Progressivo com Pesos como Resistência}

Com esse método, os exercícios destinam-se a fortalecer músculos específicos com aplicação prática do princípio da sobrecarga, constituindo a base para 
a maioria dos programas de treinamento. A realização de uma série de um exercício é menos eficiente para o aumento da força do que a realização de duas ou três séries.

O treinamento pode variar de um a cinco dias por semana, porém, o treinamento diário do mesmo grupo muscular pode impedir a boa recuperação entre as sessões de treinamento, possivelmente retardando o progresso na adaptação neuromuscular e no desenvolvimento da força. Para determinada resistência ou carga, um ritmo mais rápido do movimento pode gerar um maior aprimoramento da força.

A carga genética proporciona um arcabouço de referência diretiva que modula o efeito de cada um dos outros fatores (treinamento e nutrição) sobre o resultado final do aumento da massa muscular e da força. (MCARDLE; KATCH; KATCH, 1998 ).

O próximo tópico refere-se aos aspectos fisiológicos do treinamento de força em crianças.

\section{Aspectos Fisiológicos do Teinamento com Sobrecarga}

A ativação muscular é controlada pelo sistema nervoso. Um neurônio motor e as fibras musculares por ele enervadas constituem a unidade motora. A unidade neuromuscular é composta pelo músculo e seus nervos. A condução do impulso nervoso se dá de forma contínua ou saltatória, dependendo do tipo da fibra muscular. Apenas as unidades motoras recrutadas em um exercício produzem força, e portanto sofrerão os efeitos das mudanças adaptativas com o treinamento.

A demanda colocada sobre o músculo determina a quantidade de tecido muscular que é ativado para realizar o movimento e, assim, adapta-se ao treinamento. Se uma unidade motora for ativada, todas as fibras musculares pertencentes a ela estarão envolvidas na contração. Quanto mais unidades motoras são estimuladas em um músculo, maior é a quantidade de força desenvolvida .
A força muscular tende a aumentar em função do crescimento e da maturação da criança. Com o treinamento de sobrecarga, elas podem obter ganhos significativos na força muscular através de um aumento na eficiência de recrutamento das unidades motoras responsáveis pela contração muscular e melhora na coordenação motora, sem alterações das medidas antropométricas durante o período de treino (OLIVEIRA et al., 2003; CRESTAN et al., 2001).

Sugere-se a utilização do sistema de séries alternadas utilizado para desenvolver resistência de força. Ele consiste na execução de um exercício para um grupo muscular seguido por outro exercício para um grupo muscular em uma parte diferente do corpo (alternância de grupo musculares). Isso permite descanso parcial no grupo muscular recém treinado.

A seguir, serão apresentadas algumas orientações básicas quanto ao treinamento da força muscular em crianças de diferentes faixas etárias.

\section{Orientações Básicas para a Progressão dos Exercícios de Força para Crianças}

a) Idade de 5 a 7 anos:

— inicie a criança nos exercícios básicos com pouco ou nenhum peso;

- desenvolva o conceito de uma sessão de treinamento;

— ensine as técnicas do exercício;

— progrida de exercícios calistênicos com o peso do corpo, exercícios com parceiros e exercícios com cargas leves;

— mantenha o volume baixo.

b) Idade de 8 a 10 anos:

- aumente gradualmente o número de exercícios;

- pratique a técnica de exercício para todos os levantamentos;

— inicie incremento gradual progressivo e carga dos exercícios;

- mantenha os exercícios simples;

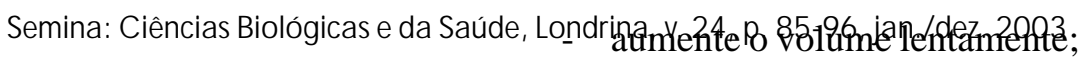


- cuidadosamente, monitore a tolerância ao estresse do exercício.

c) Idade de 11 a 13 anos:

— ensine todas as técnicas básicas dos exercícios;

- continue progressivamente aumentando o peso de cada exercício;

— enfatize a técnica do exercício;

— introduza exercícios mais avançados com pouca ou nenhuma carga.

d) Idade de 14 a 15 anos

- progrida para programas de exercícios de força mais avançados;

— inclua componentes específicos do esporte se assim desejar;

— enfatize as técnicas do exercício;

— aumente o volume.

e) Idade de 16 anos em diante:

- defina o nível inicial de programas para adultos, depois que toda a experiência anterior tenha sido adquirida (FLECK; KRAEMER, 1999).

O próximo tópico apresenta as variáveis mais importantes na elaboração dos programas de treinamento para crianças e adolescentes.

\section{Elaboração dos Programas de Treinamento}

Algumas questões precisam ser levadas em consideração, antes que a criança ou adolescente inicie um programa de treinamento de força: estar fisicamente e psicologicamente preparada para treinar; qual programa ela deve seguir; conhecer as técnicas corretas dos exercícios; os assistentes conhecem os procedimentos de segurança; a criança conhece os procedimentos de segurança; o equipamento se ajusta a criança; o programa de força é equilibrado, ou seja, a criança participa de atividades cardiovasculares e também pratica outros esportes. Cada sessão de treinamento dura de 20 à 60 minutos com a realização de três sessões por semana. $\mathrm{O}$ período de adaptação deverá ser de aproximadamente 4 semanas. Para que o programa de força, tenha sucesso deve ficar claro para os praticantes e assistentes as expectativas e objetivos realistas, evitando, por exemplo, criar a perspectiva do aumento do volume muscular em crianças que ainda não possuam maturação para tal alteração fisiológica. Cabe também alertá-las para evitarem a concorrência com outros colegas de treino, pois as adaptações ocorrem em ritmos diferentes. Deve ficar claro que o propósito do treinamento é estimular o potencial genético de cada um em relação a aptidão física. Ter equipamentos e assistentes disponíveis para desenvolver o programa. A adaptação ao programa deve ser gradativa e a avaliação para verificação dos resultados deve ser realizada não anteriormente à 8 (oito) semanas para que haja tempo para as adaptações necessárias. O registro de desempenho no treinamento deve ser registrado para efeito de pesquisa ou para simples acompanhamento na evolução do mesmo.

Para a eficiência do programa, alguns princípios precisam ser seguidos: condicionamento de todos os elementos do condicionamento físico (força, flexibilidade, capacidade cardiovascular e composição corporal); escolha de exercícios para desenvolvimento harmonioso do corpo; exercícios globais e localizados. É preciso também certificarse de que a criança terá tempo livre para brincar e desenvolver sua personalidade; não há necessidade de distinção entre meninos e meninas, pois o condicionamento físico geral requer treinamento de todos os principais grupos musculares. Com o passar do programa, deve-se enfatizar as partes mais frágeis no corpo do menino e da menina. Determinar o número de séries, repetições, intervalo e sobrecarga a ser utilizada no programa. Essas são as variáveis que irão determinar o programa de treinamento de força muscular. Para crianças de até 15 anos que visam o desenvolvimento da força muscular utilizase o seguinte programa, conforme discriminado abaixo na Tabela 1. 
Tabela 1. Determinação das variáveis no programa de força muscular em crianças

\begin{tabular}{c|c|c}
\hline Intensidade & Percentual & Repetições \\
\hline Dia intenso & $100 \%$ & de 8 a 10 RM \\
\hline Dia moderado & $90 \%$ & de 8 a 10 RM \\
\hline Dia leve & $85 \%$ & de 8 a 10 RM \\
\hline
\end{tabular}

O intervalo deve ser de 1 a 3 minutos entre as séries, dependendo da característica do treinamento. O fator chave do treinamento de força muscular é o período de recuperação e isso deverá ser levado em consideração na prescrição (freqüência, volume e intensidade). A recuperação deverá ser fisica e mental para que a criança não desista e aproveite ao máximo o programa. Uma freqüência que parece ser a ideal é de três vezes por semana tomando cuidado para não causar "overtraining", nem tornar o programa ineficaz. Deve-se discutir com a criança alguns aspectos: esforço e fadiga, dor, desconforto excessivo, modificações no programa, feedback e recuperação para a próxima sessão. Crianças prépúberes devem evitar o treinamento com carga máxima ou próxima dela, mas podem utilizar a periodização para maximizar os benefícios além de contribuir para a minimização da possibilidade de fadiga. E a periodização se dá da seguinte forma: Segunda-feira: treino leve; Quarta-feira: treino pesado; Sexta-feira: treino moderado. A variação da carga e as mudanças no formato de treinamento se dará entre 2 e 4 semanas. A Tabela 2 abaixo descreve uma proposta de periodização de treinamento em crianças pré-púberes em suas diferentes fases de treinamento, séries e repetições.
Tabela 2. Modelo de periodização para crianças prépúberes.

\begin{tabular}{c|l|l}
\hline Fase de treinamento & Séries & Repetições \\
\hline Inicial & 3 & 10 a 15 \\
\hline Força & 3 & 6 a 10 \\
\hline Potência & 2 a 3 & 6 a 8 \\
\hline Pico & 1 a 2 & 6 a 8 \\
\hline
\end{tabular}

O descanso deve ser ativo (por exemplo com realização de atividades aeróbias). Se o treinamento for direcionado à aptidão física voltada para a saúde, não haverá necessidade de treinamento de potência e visando picos de força. A variação dos exercícios pode levar um grupo muscular a elevar mais a força por ele produzida. Essa variação ou incremento de exercícios deve ser realizada entre 2 e 4 semanas de treinamento. Entretanto, é necessário manter alguns exercícios para os principais grupos musculares durante o programa de treinamento para que haja adaptações do movimento original relativas ao treinamento. Algumas variações podem ser realizadas: aumento da sobrecarga; variar o exercício para o mesmo grupo muscular; variar as repetições utilizadas (de 6 a $20 \mathrm{RM}$ ); variar a quantidade de séries (de 1 a 3); variar os dias de treino leve, pesado e moderado. Se a criança estiver necessitando de uma perda de peso corporal urgente (por excesso de gordura corporal), o programa será direcionado para esse objetivo, com a diminuição do tempo nos exercícios anaeróbicos e aumento do volume de exercícios aeróbicos. O programa sempre é direcionado à necessidade do aluno ou a aptidão física relacionada à saúde. (KRAEMER; FLECK, 2001). A seguir, são expostas algumas orientações sobre os testes de força nessa faixa etária. 


\section{Considerações Acerca dos Testes de Força Muscular em Crianças}

É essencial que todos os indivíduos participantes do treinamento sejam testados nas mesmas condições, para que possam ser feitas comparações aceitáveis entre eles. Antes do teste, fornecer instruções padronizadas antes do teste. Caso se realize aquecimento, o mesmo deve ser de duração e intensidade uniforme. $\mathrm{O}$ indivíduo deve possuir um conhecimento prático do teste (aprendizado). É preciso certificar-se de que o ângulo de mensuração sobre o membro ou dispositivo de teste seja constante entre os indivíduos. É necessário, ainda, determinar previamente, um número mínimo de ensaios (repetições) para estabelecer um escore padrão, e selecionar os testes que comportam sabidamente uma alta reprodutibilidade dos escores. A falta de fidedignidade da mensuração pode, por si só, mascarar o verdadeiro desempenho do indivíduo nesse teste. Cabe considerar as diferenças individuais em variáveis como o tamanho e a composição corporal ao avaliar os escores de força entre indivíduos e grupo. (MCARDLE; KATCH; KATCH, 1998).

Estudo realizado por Gurjão (2003) procurou analisar a quantidade de sessões necessárias para a familiarização ao teste de 1 Repetição Máxima (1$\mathrm{RM})$, em crianças pré-púberes $(9,5 \pm 0,5$ anos; $35,1 \pm 6,9 \mathrm{~kg} ; 138,3 \pm 6,1 \mathrm{~cm}$ ), do sexo masculino, sem experiência prévia em treinamento com pesos, submetidas a 8 sessões de testes nos exercícios de mesa extensora e rosca direta de bíceps, com intervalo de 48 horas entre elas. Em cada sessão, as crianças realizaram 3 tentativas intervaladas por 3-5 minutos de recuperação, para cada um dos exercícios testados. Foram encontrados aumentos de $30,2 \%$ no exercício de mesa extensora, e $22,7 \%$ no exercício de rosca direta de bíceps, ao longo das 8 sessões de testes. No entanto, nenhuma diferença significativa estatísticamente foi encontrada entre a terceira e a oitava sessão de testes na mesa extensora, e entre a quinta e a oitava sessão de testes na rosca direta de bíceps. Estes resultados indicam que a avaliação da força muscular pode ser subestimada pela falta de familiarização prévia em testes de 1-RM naquela faixa etária. Os resultados sugerem que o teste parece ser dependente da tarefa motora executada, e possivelmente, do tamanho do grupamento muscular envolvido.

Outros estudos de Faigenbaum, Miliken e Westcott (2003), Ploutz-Snyder e Giamis (2001) indicam a segurança da aplicação do teste de 1-RM em crianças pré-puberes de ambos os sexos, enfatizando a importância da familiarização na mensuração da força muscular. Um número mínimo de 3 a 4 sessões antes da avaliação da força muscular pelo teste de 1-RM foi verificada em indivíduos jovens do sexo masculino, com experiência em exercícios com pesos (CYRINO et al., 2002).

\section{Considerações Finais}

O treinamento de força muscular pode ser desenvolvido em crianças e adolescentes desde que o programa seja organizado e sistematizado para contribuir no desenvolvimento harmonioso dos movimentos e da parte estrutural de cada indivíduo. Deve-se ter muito cuidado na execução dos movimentos e na sobrecarga utilizada para cada exercício proposto, devendo a criança ser assistida por profissionais capacitados. Antes do início de um programa de treinamento com pesos para crianças, sugere-se que sejam realizados testes de 1-RM, em função da tarefa motora e tamanho do grupamento muscular envolvido, verificando-se a estabilização das medidas e aumentando a precisão da carga inicial de trabalho. $\mathrm{O}$ treinamento com sobrecarga produz um processao de melhor adaptação neuromuscular na criança e no adolescente, levando-a a um aumento significativo da força muscular e sem grandes alterações nas suas medidas antropométricas.

A musculação é mais uma opção de atividade física para crianças e adolescentes, assim como esportes, lutas, jogos, entre outros. O professor, assim com em outras áreas da Educação Física, deverá estar preparado para a atividade que irá conduzir, 
planejando o treinamento e respeitando a individualidade biológica de cada criança.

\section{Referências}

BRITO, C. F.; ANDRADE, D. R.; ARAÚJO, T. L.; MATSUDO, V. K. R. Estabilidade da aptidão física entre a infância e adolescência. Revista Brasileira de Atividade Física e Saúde, Londrina, v.4, n.1, p.711, 1999.

CRESTAN, T. A.; MAKOSKI, A.; PICHETH, D. M.; CYRINO, E. S. Adaptações neuromusculares após o treinamento com pesos. Revista Brasileira de Ciência e Movimento, Brasília, Supl., p.138, 2001.

CYRINO, E. S.; PINA, F. L. C.; PICHETH, D. M.; DIAS, R. M. R.; CARVALHO, F. O.; TASSI, G. M. et al. Influência da familiarização prévia sobre os resultados de testes de uma repetição máxima para a avaliação dos níveis de força muscular. In: CONGRESSO DE EDUCAÇÃO FÍSICA E CIÊNCIAS DO DESPORTO DOS PAIISES DE LÍNGUA PORTUGUESA, 9., 2002, São Luís. Anais... São Luís, 2002. p.179.

FAIGENBAUM, A.; MILIKEN, L. A.; WESTCOTT, W.L. Maximal strength test in healthy children. Journal of Strength and Conditioning Research, Colorado Springs, n.17, p.162-166, 2003.

FIORESE, L. Os efeitos do treinamento precoce em crianças e adolescentes. Revista da Fundação de Esporte e Turismo, Curitiba, v.1, n.2, p.23-31, 1989.

FLECK, S. Riscos e benefícios do treinamento de força em crianças: novas tendências. Revista Brasileira de Atividade Física e Saúde, Londrina, v.2, n.1, p.69-75, 1997.

FLECK, S. J.; KRAEMER, W. J. Fundamentos do Treinamento de Força Muscular. Porto Alegre: Artes Médicas Sul, 1999.

GUEDES, D. P.; GUEDES, J. E. R. P. Aptidão física relacionada à saúde de crianças e adolescentes: avaliação referenciada por critério. Revista Brasileira de Atividade Física e Saúde, Londrina, v.1, n.2, p.27-38, 1995.

Exercício Físico na Promoção da Saúde. Londrina: Midiograf, 1995.

GURJÃO, A. L D. A influência do processo de familiarização na avaliação da força muscular em crianças pré-púberes. Trabalho de Conclusão de Curso (Graduação em Educação Física) - Universidade Estadual de Londrina, 2003.

LOPES, A. G. Possíveis alterações epifisiárias em função do treinamento de força muscular em pré-púberes. Trabalho de Conclusão de Curso (Graduação em Educação
Física) - Universidade Estadual de Londrina, Paraná. Disponível em: 〈http://www.boletimef.org/default.asp〉. Acesso em: 10 set. 2003.

McARDLE, W. D.; KATCH, F. I.; KATCH, V. L. Fisiologia do Exercício: energia, nutrição e desempenho humano. 4.ed. Rio de Janeiro: Guanabara Koogan, 1998.

OLIVEIRA, A. R. Fatores influenciadores na determinação do nível de aptidão física em crianças. Synopsis, Curitiba, v.7, p.48-62, 1996.

OLIVEIRA, A. R.; CYRINO, E. S.; FREITAS, L. A. G.; RISSO, S.; LOPES, A. G.; MACHADO, M. V.; OLIVEIRA, F. C. A adaptação neuromuscular no treinamento com sobrecarga em crianças. In: CONGRESSO INTERNACIONAL DE PEDAGOGIA DO ESPORTE, 1. 2003, Maringá. Anais... Maringá, 2003. p. 24, 27, 43.

OLIVEIRA, A. R.; GALLAGHER, J. D. The influence of sport experience and selective attention on the development of balance control. In: NORTH AMERICAN SOCIETY OFPSYCHOLOGY OF SPORT AND PHYSICAL ACTIVITY ANNUAL CONFERENCE (NASPSPA), 1999, Clearwater Beach. Annals... Clearwater Beach, 1999.

. Treinamento de força em crianças de 6 a 12 anos de idade: um estudo em meta análise. In: SIMPÓSIO INTERNACIONALDECIÊNCIAS DOESPORTE, 21., 1998, São Paulo. Anais...São Paulo, 1998. p.8, 11, 120.

Treinamento de força muscular em crianças: novas tendências. Revista Brasileira de Atividade Física e Saúde, Londrina, v.2, n.3, p.80-90, 1997.

Strength training in children: a meta analysis. Pediatric Exercise Science, Champaign, v.7, n.1, p.108, feb. 1995.

OLIVEIRA, A. R. de; GALLAGHER, J. D.; SILVA, S. G. Musculação em crianças: uma meta análise. Revista da Associação dos Professores de Educação Física de Londrina, Londrina, v.10, n.18, p.70-76, 1995.

PINHO, R. A.; PETROSKI, E. L. Nível de atividade física em crianças. Revista Brasileira de Atividade Física e Saúde, Londrina, v.2, n.3, p.67-79, 1997.

PLOUTZ-SNYDER, L. L.; GIAMIS, E. L. Orientation and familiarization to 1-RM strength testing in old and young women. Journal of Strength and Conditioning Research, Colorado Springs, n.15, p.519-523, 2001.

RAMOS, A. T. Criança/adolescente e a atividade física. Revista Técnica de Educação Física e Desportos, Rio de Janeiro, v 17, n 94, p.1-2, 1998.

RISSO, S.; LOPES, A. G.; OLIVEIRA, A. R. Repensando o treinamento da força muscular em crianças pré-púberes na faixa etária de 6 a 12 anos de idade. Revista Treinamento Desportivo, Londrina, v.4, n.1, p.48-54, 1999. 
ROBERTS, S.; WEIDER, B. Strength and Weight Training for Young Athletes. Chicago: Contemporary Books, 1994.

ROCHA, P. S. O. Treinamento Desportivo I. Brasília: Ministério da Educação e Cultura, 1978.

\section{Agradecimentos pela elaboração do artigo às} instituições:

Universidade Estadual de Londrina - UEL;

Grupo de Estudo e Pesquisa em Metabolismo, Nutrição e Exercício - GEPEMENE;

Conselho Nacional de Desenvolvimento Científico e Tecnológico - CNPq. 